\title{
ON THE HOMOTOPY TYPE OF ANR'S
}

\author{
J. H. C. WHITEHEAD
}

1. Introduction. If $X$ and $Y$ are any spaces and if $f: X \rightarrow Y$ and $g: Y \rightarrow X$ are maps such that $g f \simeq 1$, then $g$ is called a left homotopy inverse of $f$ and $f$ a right homotopy inverse ${ }^{1}$ of $g$. In this case we shall say that $Y$ dominates $^{2} X$. If $Y$ dominates $X$ and $Z$ dominates $Y$ then it is easily verified that $Z$ dominates $X$. If $g$ is both a right and left homotopy inverse of $f$ it is called a homotopy inverse of $f$ and $f$ will be called a homotopy equivalence. Thus the assertion that $f: X \rightarrow Y$ is a homotopy equivalence claims that $X$ and $Y$ are of the same homotopy type and, moreover, that $f$ has a homotopy inverse.

Two maps, $f_{0}, f_{1}: X \rightarrow Y$ are said (cf. [1, pp. 49, 50] and [2, p. 344]) to be $n$-homotopic if, and only if, $f_{0} \phi \simeq f_{1} \phi$ for every map, $\phi: P \rightarrow X$, of every (finite) polyhedron, $P$, of at most $n$ dimensions. By an $n$-homotopy inverse of a map, $f: X \rightarrow Y$, or an n-homotopy equivalence we mean the same as a homotopy inverse or a homotopy equivalence with homotopy replaced by $n$-homotopy throughout the definition.

By a CR-space we shall mean a connected compactum, which is an ANR (absolute neighborhood retract). Any CR-space, $X$, is dominated by a finite simplicial complex [5, Theorems $12.2,16.2$, pp. 93, 99], even if its dimensionality is infinite. We shall use $\Delta X$ to denote the minimum dimensionality of all (finite, simplicial) complexes which dominate $X$. Then $\Delta X \leqq \operatorname{dim} X$ and we may think of $\Delta X$ as a kind of "quasi-dimensionality," noticing, however, that $\Delta X$ may be less than $\operatorname{dim} X$, even if $X$ is itself a finite polyhedron.

Let $X, Y$ be CR-spaces, and let $N=\max (\Delta X, \Delta Y)$. Let $f: X \rightarrow Y$ be a given map and let $f_{n}: \pi_{n}(X) \rightarrow \pi_{n}(Y)$ be the homomorphism induced by $f$. If $f$ is a homotopy equivalence then $f_{n}$ is an isomorphism onto for each $n \geqq 1$. In $\S 3$ below we prove a sharper theorem than the converse, namely:

THEOREM 1. If $f_{n}: \pi_{n}(X) \rightarrow \pi_{n}(Y)$ is an isomorphism onto for each $n=1, \cdots, N$, then $f: X \rightarrow Y$ is a homotopy equivalence. ${ }^{3}$

Received by the editors January $26,1948$.

${ }_{1} \mathrm{Cf}$. [1]. Numbers in brackets refer to the references cited at the end of the paper.

${ }^{2}$ In this case the homomorphisms $H_{n}(Y) \rightarrow H_{n}(X)$ induced by $g: Y \rightarrow X$ are all onto, likewise the induced homomorphisms $\pi_{n}(Y) \rightarrow \pi_{n}(X)$, assuming $X, Y$ to be arcwise connected. In fact $H_{n}(Y)$, or $\pi_{n}(Y)(n \geqq 2)$, may be represented as the direct sum of $H_{n}(X)$, or $\pi_{n}(X)$, and the kernel of this homomorphism.

${ }^{3}$ If $X$ and $Y$ are of the same homotopy type, then each dominates the other and $\Delta X=\Delta Y$. Theorem 1 is formulated with a view to applications in which it is possible to calculate separate upper bounds for $\Delta X, \Delta Y$ (for example, $\operatorname{dim} X, \operatorname{dim} Y$ ). 
We shall also prove:

THEOREM 2. The map $f: X \rightarrow Y$ is an $(N-1)$-homotopy equivalence if, and only if, $f_{n}: \pi_{n}(X) \rightarrow \pi_{n}(Y)$ is an isomorphism onto for each $n=1, \cdots, N-1$.

If $\Delta X=\Delta Y=0$ then it is obvious that $X$ and $Y$, being connected, are both absolute retracts. Therefore any map, $X \rightarrow Y$, is a homotopy equivalence and Theorem 1, likewise Theorem 3 below, is trivial. Similarly Theorems 2 and 4 are trivial if $N \leqq 1$. Therefore we shall assume that $N \geqq 1$ in Theorems 1 and 3 and $N \geqq 2$ in Theorems 2 and 4 .

Theorem 2 is significant in the theory of polyhedra or cell complexes. For the $(n-1)$-homotopy type of the $n$-section is a homotopy invariant of a given complex $K$ (that is, is the same for any complex of the same homotopy type). It is equivalent to what I have previously called the $n$-group (see [6] and [7]) of $K$, but now propose to call the $n$-type. These statements will be proved in a later paper in which the $n$-type of a complex will be further discussed.

A map $f: X \rightarrow Y$ is not necessarily an $m$-homotopy equivalence if $f_{1}, \cdots, f_{m}$ are isomorphisms onto, where $m<N-1$. For example, let $Y$ be a complex projective plane, let $X \subset Y$ be a 2 -sphere, which is a complex line in $Y$, and let $f: X \rightarrow Y$ be the identity. If $g: Y \rightarrow X$ were a 2-homotopy inverse of $f$, then $g \mid X=g f: X \rightarrow X$ would be of degree +1 and would therefore induce the identical automorphism of $\pi_{3}(X)$. But $f_{3} \pi_{3}(X)=0$. Therefore it would be absurd to suppose that $g \mid X$ can be extended to a map $g: Y \rightarrow X$.

Theorem 1, restricted to polyhedra and weakened by replacing $N$ by $\max (\operatorname{dim} X+1, \operatorname{dim} Y)$, is essentially a restatement of parts of Theorems 15 and 17 in [6, pp. 273 and 277]. The generalization to CR-spaces was suggested by a theorem proved by Sze-Tsen $\mathrm{Hu}$ in [9]. However we do not actually use Hu's theorem. Instead we follow Lefschetz's approach to the subject and eventually deduce Hu's theorem in a modified form. Of course Theorem 1 does not mean that $X$ and $Y$ are necessarily of the same homotopy type if $\pi_{n}(X)$ $\approx \pi_{n}(Y)$ for all values ${ }^{4}$ of $n$. The crux of the matter is not merely that $\pi_{n}(X) \approx \pi_{n}(Y)$ but that a certain set of isomorphisms, $f_{n}: \pi_{n}(X)$ $\rightarrow \pi_{n}(Y)$, can be "realized geometrically" by means of a map $f: X \rightarrow Y$.

Let $\tilde{X}$ be the universal covering space of a given CR-space, $X$, with base point $x_{0} \in X$. Then a point, $\tilde{x} \in \tilde{X}$, is a homotopy class of

\footnotetext{
${ }^{4}$ For example $\pi_{n}\left(P^{4} \times S^{3}\right) \approx \pi_{n}\left(S^{5} \times S^{2}\right)$ for every $n \geqq 1$, where $P^{4}$ is a complex projective plane and $S^{r}$ is an $r$-sphere. This example is due to Hsien-Chung Wang. For other examples see [10].
} 
paths, with fixed end points, joining $x_{0}$ to the point $p \tilde{x} \in X$, where $p$ is the projection, $p: \tilde{X} \rightarrow X$, which is thus defined. Thus $\tilde{X}$ contains a base point, $\tilde{x}_{0} \in \tilde{X}$, which corresponds to the constant path on $x_{0}$. If $\pi_{1}(X)=1$ we identify $\tilde{X}$ with $X$, taking $\tilde{x}=p \tilde{x}$. Let $H_{n}(\tilde{X})$ be the $n$th homology group ${ }^{5}$ of $\tilde{X}$. Let $Y$ be another CR-space, let $\tilde{Y}$, $H_{n}(\tilde{Y})$ be similarly defined and let $\boldsymbol{p}$ also denote the projection $p: \hat{Y} \rightarrow Y$. Then a given map $f: X \rightarrow Y$ can be "lifted" into a unique map $\tilde{f}: \tilde{X} \rightarrow \tilde{Y}$, such that $f \boldsymbol{p}=p \tilde{f}, \tilde{f} \tilde{x}_{0}=\tilde{y}_{0}$, where $y_{0}=f x_{0}$ and $\tilde{y}_{0}$ are the base points in $Y$ and $\tilde{Y}$. The map $\tilde{f}$ induces homomorphisms $H_{n}(\tilde{X})$ $\rightarrow H_{n}(\tilde{Y})$, which we shall also describe as induced by $f: X \rightarrow Y$. In particular $Y$ may be a finite, $\Delta X$-dimensional polyhedron, which dominates $X$, and $f: X \rightarrow Y$ a map with a left inverse $g: Y \rightarrow X$. Let $\tilde{g}: \tilde{Y} \rightarrow \tilde{X}$ be the map obtained by lifting $g$. Then a homotopy $g f \rightarrow 1$ may be lifted into a homotopy $\tilde{g} \tilde{f} \rightarrow u$, where $u: \tilde{X} \rightarrow \tilde{X}$ is a transformation in the covering group (that is, $p u=p$ ). Therefore $u^{-1} \tilde{g}$ is a left homotopy inverse of $\tilde{f}$ (likewise $\tilde{f} u^{-1}$ is a right homotopy inverse of $\tilde{g}$ ) and $\tilde{Y}$ dominates $\tilde{X}$. It follows that $H_{n}(\tilde{X})=0$ if $n>\operatorname{dim} \tilde{Y}=\operatorname{dim} Y=\Delta X$. Therefore, if $X, Y$ are any two CR-spaces, $H_{n}(\tilde{X})=0, H_{n}(\tilde{Y})=0$ if $n>\max (\Delta X, \Delta Y)$. We shall prove:

Theorem 3. If $X, Y$ are any $C R$-spaces, then a map $f: X \rightarrow Y$ is a homotopy equivalence if each of the induced homomorphisms $f_{1}: \pi_{1}(X)$ $\rightarrow \pi_{1}(Y), H_{n}(\widetilde{X}) \rightarrow H_{n}(\widetilde{Y})(n=2,3, \cdots)$ is an isomorphism onto.

As a corollary to this we have:

Corollary 1. If $X, Y$ are simply connected $C R$-spaces, then a map $f: X \rightarrow Y$ is a homotopy equivalence if each of the induced homomorphisms $H_{n}(X) \rightarrow H_{n}(Y)(n=2,3, \cdots)$ is an isomorphism onto.

Let $X$ be a finite cell complex. ${ }^{6}$ Then the groups $H_{n}(\tilde{X})$ may be defined in terms of chain groups, ${ }^{7} C_{n}(\tilde{X})$, which are free $\Re(X)$ modules, where $\Re(X)$ is the group ring of $\pi_{1}(X)$, with integral coefficients. According to Eilenberg and Steenrod a map, $f: X \rightarrow Y$, of $X$ into another complex $Y$, is said to be cellular if, and only if, $f X^{n} \subset Y^{n}$ for each $n=0,1, \cdots$, where $X^{n}, Y^{n}$ are the $n$-sections of

${ }^{5}$ It is to be understood that all our homology groups are defined, as in [12], in terms of singular chains with integral coefficients.

- That is, a complex of the sort defined on p. 1235 of [7] or in a forthcoming book by S. Eilenberg and N. E. Steenrod.

${ }^{7}$ Cf. [11, chap IV, \$17]. The generalization from ordinary polyhedral complexes to the more general cell complexes will be described in the book by Eilenberg and Steenrod. $C_{n}(\widetilde{X})$ is the relative homology group $H_{n}\left(\widetilde{X}^{n}, \widetilde{X}^{n-1}\right)$, where $\widetilde{X}^{r}$ is the $r$-section of $\widetilde{X}$. For an account of chain mappings and chain equivalences see [4] and [12]. 
$X, Y$. A cellular map, $f: X \rightarrow Y$, determines a chain mapping, $\gamma: C_{n}(\tilde{X})$ $\rightarrow C_{n}(\tilde{Y})$, which is an operator homomorphism, for each $n=0,1, \cdots$, in the sense that $\gamma(\rho c)=(\alpha \rho) \gamma c$, where $\rho \in \Re(X), c \in C_{n}(\tilde{X})$ and $\alpha: \Re(X) \rightarrow \Re(Y)$ is the homomorphism induced by $f_{1}: \pi_{1}(X) \rightarrow \pi_{1}(Y)$.

A chain mapping $\gamma: C(\tilde{X}) \rightarrow C(\tilde{Y})$ of the family $C(\tilde{X})=\left\{C_{n}(\tilde{X})\right\}$ into the family $C(\tilde{Y})=\left\{C_{n}(\tilde{Y})\right\}$ is defined in purely algebraical terms as a homomorphism, $\alpha: \pi_{1}(X) \rightarrow \pi_{1}(Y)$, together with a family of operator homomorphisms, $\gamma: C_{n}(\tilde{X}) \rightarrow C_{n}(\tilde{Y})$, such that $\partial \gamma=\gamma \partial$, where $\partial$ is the boundary operator. If $\gamma$ is the chain mapping, which is induced by some (cellular) map $f: X \rightarrow Y$, then $f$ will be described as a geometrical realization of $\gamma$. From Theorem 2 we have the corollary:

COROLlaRY 2. If a given chain equivalence $\gamma: C(\tilde{X}) \rightarrow C(\tilde{Y})$ has a geometrical realization, $f: X \rightarrow Y$, then $f$ is a homotopy equivalence.

This corollary shows that the problem of determining conditions for a given chain mapping to have a geometrical realization is fundamental in the homotopy theory of complexes. In a later paper we shall prove that, if $X$ is at most 3-dimensional, then any chain mapping, $\gamma: C(\tilde{X}) \rightarrow C(\tilde{Y})$, has a geometrical realization, subject to certain conditions on $\gamma: C_{0}(\tilde{X}) \rightarrow C_{0}(\tilde{Y})$.

Let $X, Y, f: X \rightarrow Y$ be as in Theorem 2 and let $N=\max (\Delta X, \Delta Y)$. Then we prove, as a companion to Theorem 2:

THEOREM 4. The map $f: X \rightarrow Y$ is an $(N-1)$-homotopy equivalence if

(a) each of the induced homomorphisms $f_{1}: \pi_{1}(X) \rightarrow \pi_{1}(Y), H_{n}(\tilde{X})$ $\rightarrow H_{n}(\tilde{Y})(n=0, \cdots, N-2)$ is an isomorphism onto,

(b) the induced homomorphism $H_{N-1}(\tilde{X}) \rightarrow H_{N-1}(\tilde{Y})$ is onto,

(c) $f_{N-1}: \pi_{N-1}(X) \rightarrow \pi_{N-1}(Y)$ is an isomorphism into.

Conversely, if $f: X \rightarrow Y$ is an $(N-1)$-homotopy equivalence, so is the lifted map, $\tilde{f}: \tilde{X} \rightarrow \tilde{Y}$, and $H_{n}(\tilde{X}) \rightarrow H_{n}(\tilde{Y})$ is an isomorphism onto for $n=0,1, \cdots, N-1$.

2. A lemma on mapping cylinders. Let $A, B$ be any two spaces and $A_{0} \subset A, B_{0} \subset B$ any subsets of $A, B$. We shall say that the pair $\left(B, B_{0}\right)$ dominates $\left(A, A_{0}\right)$ if, and only if, there are maps, $f:\left(A, A_{0}\right) \rightarrow\left(B, B_{0}\right)$ and $g:\left(B, B_{0}\right) \rightarrow\left(A, A_{0}\right)$, such that $g f$ is deformable into the identity by a homotopy of the form $\xi_{t}:\left(A, A_{0}\right) \rightarrow\left(A, A_{0}\right)$.

Let $X, Y$ be any compacta and $f: X \rightarrow Y$ a given map. We form the topological product $X X I$ and, replacing $X$ by a homeomorph, if necessary, assume that no two of $X, Y, X \times I$ have a point in common.

\footnotetext{
${ }^{8}$ It is to be understood that the homomorphism $f_{1}: \pi_{1}(X) \rightarrow \pi_{1}(Y)$ associated with a chain equivalence is an automorphism onto.
} 
Let $Z$ be the mapping cylinder, which is formed by identifying ${ }^{9}$ $(x, 0) \in X \times I$ with $x$ and $(x, 1)$ with $f x \in Y$ for each $x \in X$. Let $P, Q$ be compacta, which dominate $X, Y$, respectively, and let $\lambda: X \rightarrow P$, $\lambda^{\prime}: P \rightarrow X, \mu: Y \rightarrow Q, \mu^{\prime}: Q \rightarrow Y$ be maps such that $\lambda^{\prime} \lambda \simeq 1, \mu^{\prime} \mu \simeq 1$. Let $R$ be the mapping cylinder of the map $\mu f \lambda^{\prime}: P \rightarrow Q$. Then our lemma is:

LEMMA 1. The pair $(R, P)$ dominates $(Z, X)$.

Let $\xi_{t}: X \rightarrow X$ and $\eta_{t}: Y \rightarrow Y$ be homotopies such that $\xi_{0}=\lambda^{\prime} \lambda, \xi_{1}=1$, $\eta_{0}=\mu^{\prime} \mu, \eta_{1}=1$ and let $\nu:(Z, X) \rightarrow(R, P)$ be given by

$$
\begin{aligned}
\nu(x, t) & =(\lambda x, 2 t) & & (\text { if } 0 \leqq 2 t \leqq 1) \\
& =\mu f \xi_{2 t-1} x & & (\text { if } 1 \leqq 2 t \leqq 2), \\
\nu y & =\mu y & & (x \in X, y \in Y) .
\end{aligned}
$$

This is single-valued, hence continuous (see $[8, \S 5]$ ), since $(\lambda x, 1)$ $=\left(\mu f \lambda^{\prime}\right) \lambda x=\mu f \xi_{0} x, \mu f \xi_{1} x=\mu f x$. Let $\nu^{\prime}:(R, P) \rightarrow(Z, X)$ be given by

$$
\begin{aligned}
\nu^{\prime}(p, t) & =\left(\lambda^{\prime} p, 2 t\right) & & (\text { if } 0 \leqq 2 t \leqq 1) \\
& =\eta_{2-2 t} f \lambda^{\prime} p & & (\text { if } 1 \leqq 2 t \leqq 2), \\
\nu^{\prime} q & =\mu^{\prime} q & & (p \in P, q \in Q) .
\end{aligned}
$$

This is single-valued since $\left(\lambda^{\prime} p, 1\right)=f \lambda^{\prime} p=\eta_{1} f \lambda^{\prime} p$ and $\eta_{0} f \lambda^{\prime} p=\mu^{\prime}$ $\cdot\left(\mu f \lambda^{\prime}\right) p$. The map $\nu^{\prime} \nu:(Z, X) \rightarrow(Z, X)$ is given by

$$
\begin{aligned}
\nu^{\prime} \nu(x, t) & =\nu^{\prime}(\lambda x, 2 t) & & (\text { if } 0 \leqq 2 t \leqq 1) \\
& =\nu^{\prime} \mu f \xi_{2 t-1} x & & (\text { if } 1 \leqq 2 t \leqq 2), \\
\nu^{\prime} \nu y & =\nu^{\prime} \mu y, & &
\end{aligned}
$$

or by

$$
\begin{aligned}
\nu^{\prime} \nu(x, t) & =\left(\lambda^{\prime} \lambda x, 4 t\right) & & (\text { if } 0 \leqq 4 t \leqq 1) \\
& =\eta_{2-4 t} f \lambda^{\prime} \lambda x & & (\text { if } 1 \leqq 4 t \leqq 2) \\
& =\mu^{\prime} \mu f \xi_{2 t-1} x & & (\text { if } 1 \leqq 2 t \leqq 1), \\
\nu^{\prime} \nu y & =\mu^{\prime} \mu y . & &
\end{aligned}
$$

The desired homotopy, $\zeta_{s}:(Z, X) \rightarrow(Z, X)$, is given by

$$
\begin{aligned}
\zeta_{s}(x, t) & =\left(\xi_{s} x,(4-3 s) t\right) & (\text { if } 0 \leqq t \leqq 1 /(4-3 s)) \\
& =\eta_{2-(4-3 s) t} f \xi_{s} x & (\text { if } 1 /(4-3 s) \leqq t \leqq(2-s) /(4-3 s)) \\
& =\eta_{s} f \xi_{\rho(s, t)} x & (\text { if }(2-s) /(4-3 s) \leqq t \leqq 1), \\
\zeta_{s} y & =\eta_{s} y, &
\end{aligned}
$$

${ }^{9}$ The points in $X, Y$ shall retain their individualities in $Z$, so that $X, Y \subset Z$. 
where $\rho(s, t)=\{(4-3 s) t+3 s-2\} / 2$. It is easy to verify that $\zeta_{s}$ is single-valued and that $\zeta_{0}=\nu^{\prime} \nu, \zeta_{1}=1$. Moreover $\zeta_{s} x=\xi_{s} x \in X$ if $x=(x, 0) \in X$. Therefore $(R, P)$ dominates $(Z, X)$.

3. Proof of Theorem 1. Let $X, Y$ and $f: X \rightarrow Y$ satisfy the conditions of Theorem 1 and let $Z$ be the mapping cylinder of $f$. The theorem will follow from [1, Theorem 3.7, p. 45] (see also [3]), when we have proved that $X$ is a deformation retract of $Z$.

Let $g_{s}: Z \rightarrow Z$ be the deformation which is given by $g_{s} \mid Y=1$, $g_{s}(x, t)=(x, s+t-s t)(0 \leqq s \leqq 1)$. Then $g_{0}=1, g_{1} Z=Y$ and $g_{1} x=f x$ for each $x=(x, 0) \in X$. Let $k: Z \rightarrow Y$ be the map which is given ${ }^{10}$ by $k z=g_{1} z$ for each $z \in Z$. Then $g_{1}=j k, f=k i$, where $i, j$ are the identical maps $i: X \rightarrow Z, j: Y \rightarrow Z$. Let $\pi_{n}(X), \pi_{n}(Z), \pi_{n}(Z, X)$ be referred to a base point $x_{0} \in X$, and $\pi_{n}(Y)$ to $f x_{0} \in Y$ as base point. Let $i_{n}: \pi_{n}(X)$ $\rightarrow \pi_{n}(Z), k_{n}: \pi_{n}(Z) \rightarrow \pi_{n}(Y)$ be the homomorphisms induced by $i, k$ and let $j_{n}: \pi_{n}(Y) \rightarrow \pi_{n}(Z)$ be the homomorphism induced by $j$ and the segment (cf. [13] and [6, pp. 279 et seq.]) $\left(x_{0}, I\right)$, which joins $x_{0}$ to $f x_{0}$. Since $k j=1: Y \rightarrow Y, j k=g_{1} \simeq 1: Z \rightarrow Z$ and since $g_{s} x_{0}$ travels along the segment $\left(x_{0}, I\right)$ in the homotopy $g_{s}$, it follows that $j_{n}$ is an isomorphism onto and that $k_{n}$ is its inverse. Since $f=k i$ we have $f_{n}=k_{n} i_{n}$. Therefore $i_{n}=j_{n} f_{n}$ and $i_{n}$, like $f_{n}$, is an isomorphism onto for $n=1, \cdots, N$.

Let $2 \leqq n \leqq N$ and consider the homotopy sequence

$$
\pi_{n}(X) \stackrel{1}{\rightarrow} \pi_{n}(Z) \stackrel{2}{\rightarrow} \pi_{n}(Z, X) \stackrel{3}{\rightarrow} \pi_{n-1}(X) \stackrel{4}{\rightarrow} \pi_{n-1}(Z),
$$

in which (1) is $i_{n}$ and (4) is $i_{n-1}$. Since (1) is onto it follows from the exactness of the sequence that (2) maps $\pi_{n}(Z)$ into zero. Since (4) is an isomorphism it follows that (3) is into zero and (2) is onto. Therefore $\pi_{n}(Z, X)=0$ for $n=1, \cdots, N$, where $\pi_{1}(Z, X)=0$ means that ${ }^{11}$ $i_{1}: \pi_{1}(X) \rightarrow \pi_{1}(Z)$ is onto. Notice that if, in addition, $i_{N+1}$ is onto, then it follows from (3.1) that $\pi_{N+1}(Z, X)=0$.

Let $P$ be a finite, $\Delta X$-dimensional simplicial complex, which dominates $X$, and $Q$ a finite, $\Delta Y$-dimensional simplicial complex which dominates $Y$. Let $R, \lambda, \mu, \eta_{t}$, etc. mean the same as in $\S 2$. Since $Z$ is (obviously) arcwise connected and since $\pi_{n}(Z, X)=0$ for $n$ $=1, \cdots, N \geqq \operatorname{dim} Q$ it follows from a standard argument ${ }^{12}$ that

${ }^{10}$ We distinguish between maps $u: A \rightarrow B, v: A \rightarrow C$, where $B C C, B \neq C$, even if $u a=v a$ for each $a \in A$.

${ }^{11}$ Since $X$ is arcwise connected this is equivalent to the condition that any arc in $Z$, with its end points in $X$, is deformable, with its end points held fixed, into an arc in $X$.

$12[14$, p. 526]. This argument is recapitulated, in a slightly more general form, in $\$ 8$ below (Lemma 5). 
there is a homotopy, $\delta_{t}: Q \rightarrow Z$, such that $\delta_{0}=\nu^{\prime} \mid Q=j \mu^{\prime}, \delta_{1} Q \subset X$. Therefore $\delta_{t} \mu: Y \rightarrow Z$ is a homotopy such that $\delta_{0} \mu=j \mu^{\prime} \mu=j \eta_{0}, \delta_{1} \mu Y$ $\subset \delta_{1} Q \subset X$. Moreover we assume, as we obviously may, that $\delta_{1} \mu f x_{0}=x_{0}$, where $x_{0}$ is the base point of $\pi_{n}(X)$ and $\pi_{n}(Z)$. Let $h_{t}: Y \rightarrow Z$ be the homotopy which is given by

$$
\begin{array}{rlrl}
h_{t} & =j \eta_{1-2 t} & & \text { (if } 0 \leqq 2 t \leqq 1) \\
& =\delta_{2 t-1} \mu & \text { (if } 1 \leqq 2 t \leqq 2) .
\end{array}
$$

Then $h_{0}=j, h_{1} Y \subset X, h_{1} x_{0}=x_{0}$. Therefore the resultant of the homotopy $g_{t}: Z \rightarrow Z$, followed by $h_{t} k: Z \rightarrow Z$, is a deformation, $\Delta_{t}: Z \rightarrow Z$, such that $\Delta_{0}=1, \Delta_{1} Z \subset X, \Delta_{1} x_{0}=x_{0}$.

Let $c \in \pi_{1}(Z)$ be the element which is represented by the track of $x_{0}$ in the homotopy $\Delta_{t}$. Since $i_{1}$ is onto we have $c=i_{1} a$ for some $a \in \pi_{1}(X)$. Let $\theta_{0}:\left(S^{n}, p_{0}\right) \rightarrow\left(Z, x_{0}\right)$ be a map representing a given element $\gamma \in \pi_{n}(Z)(n \geqq 2)$, where $p_{0}$ is the base point in the standard $n$-sphere $S^{n}$. Then $\theta_{t}=\Delta_{t} \theta_{0}$ is a deformation of $\theta_{0}$ into the map $\theta_{1}=i \theta$, where $\theta:\left(S^{n}, p_{0}\right) \rightarrow\left(X, x_{0}\right)$ is given by $\theta p=\Delta_{1} \theta_{0} p\left(p \in S^{n}\right)$. Therefore

$$
\gamma=c i_{n} \alpha=\left(i_{1} a\right)\left(i_{n} \alpha\right)=i_{n}(a \alpha),
$$

where $\alpha \in \pi_{n}(X)$ is the element represented by $\theta$ and $c i_{n} \alpha, a \alpha$ are the images of $i_{n} \alpha, \alpha$ in the automorphisms ([13] and [6, pp. 279 et seq. $]$ ) determined by $c, a$. Hence $i_{n}$ is onto for every $n \geqq 1$. Taking $n=N+1$, it follows from (3.1) that $\pi_{N+1}(Z, X)=0$.

It follows from an extension of a theorem due to Borsuk ([15] and [8]) that $Z$ is an ANR. Therefore the homotopy, $\delta_{t}: Q \rightarrow Z$, defined above, can be extended, first throughout $P \cup Q$ by defining $\delta_{t} p$ $=\nu^{\prime} p \in X$ if $p \in P$, and then to a homotopy $\delta_{t}^{\prime}: R \rightarrow Z$, such that $\delta_{0}^{\prime}=\nu^{\prime}$. Since $\pi_{n}(Z, X)=0$ if $1 \leqq n \leqq N+1$ and since $N+1 \geqq \operatorname{dim} R$, it follows from repeated applications of Lemma 5 , in $\$ 8$ below (cf. the proof of Lemma 6), that there is a homotopy, $\rho_{t}: R \rightarrow Z$, rel. $P$ (that is, $\rho_{t}\left|P=\rho_{0}\right| P$ ), such that $\rho_{0}=\delta_{1}^{\prime}, \rho_{1} R \subset X$. The resultant of $\delta_{t}^{\prime}$, followed by $\rho_{t}$, is a homotopy, $\phi_{t}: R \rightarrow Z$, rel. $P$, such that $\phi_{0}=\nu^{\prime}, \phi_{1} R$ $C X$. Then $\phi_{t} \nu: Z \rightarrow Z$ is a homotopy such that $\phi_{0} \nu=\nu^{\prime} \nu=\zeta_{0}, \phi_{1} \nu Z$ $C \phi_{1} R \subset X$. Therefore the resultant of $\zeta_{1-t}:(Z, X) \rightarrow(Z, X)$, followed by $\phi_{t} \nu$, is a homotopy, $\psi_{t}:(Z, X) \rightarrow(Z, X)$, such that $\psi_{0}=1, \psi_{1} Z \subset X$. It follows from [1, Theorem 1.4, p. 42], and [3] that $X$ is a deformation retract of $Z$ and the proof is complete.

Notice that Theorem 1 follows more directly on the alternative hypothesis that $f_{n}: \pi_{n}(X) \rightarrow \pi_{n}(Y)$ is an isomorphism onto for $n$ $=1, \cdots, m-1$ and $f_{m}$ is onto, where $m=\max (\Delta X+1, \Delta Y)=\operatorname{dim} R$. For in this case it follows from (3.1) that $\pi_{n}(Z, X)=0$ for $n=1, \cdots, m$ and the paragraph showing that $\pi_{N+1}(Z, X)=0$ is unnecessary. 
Let $Z$ and $X \subset Z$ be CR-spaces and let $\pi_{n}(Z, X)=0$ for $n=1, \cdots, m$ $=\max (\Delta X+1, \Delta Z)$. Then it follows from the homotopy sequence (3.1) that $i_{n}: \pi_{n}(X) \rightarrow \pi_{n}(Z)$ is an isomorphism onto for $n=1, \cdots$, $m-1$ and that $i_{m}$ is onto. Therefore the identity map $i: X \rightarrow Z$ is a homotopy equivalence, whence $X$ is a deformation retract of $Z$. Hence we have the modified form of Hu's generalization of Hurewicz's theorem ([14, Theorem IV, p. 522] and [9]):

Corollary ${ }^{13}$ If $\pi_{n}(Z, X)=0$ for $n=1, \cdots, \max (\Delta X+1, \Delta Z)$, then $X$ is a deformation retract of $Z$.

4. Proof of Theorem 2. Let $f_{n}: \pi_{n}(X) \rightarrow \pi_{n}(Y)$ be an isomorphism onto for $n=1, \cdots, N-1$. Using the same notation as in $\S 3$, we shall prove that $i: X \rightarrow Z$ is an $(N-1)$-homotopy equivalence. Since $k: Z \rightarrow Y$ is a homotopy equivalence and $f=k i$ it will then follow that $f$ is an $(N-1)$-homotopy equivalence. It follows from (3.1) that $\pi_{n}(Z, X)=0$ for $n=1, \cdots, N-1$. Therefore there is a homotopy, $\delta_{t}: Q \rightarrow Z$, such that $\delta_{0}=j \mu^{\prime}, \delta_{1} Q^{N-1} \subset X$, where $Q^{n}$ is the $n$-section of $Q$. Since $i_{N-1}$ is an isomorphism it follows from an argument which is similar to one used in proving Lemma 6 , in $\$ 8$ below, that $\delta_{1} \mid Q^{N-1}$ can be extended to a map $i \theta$, where $\theta$ is of the form $\theta: Q \rightarrow X$. Then $i \theta\left|Q^{N-1} \simeq j \mu^{\prime}\right| Q^{N-1}$. Since $\theta, \mu, k$ are of the form $k: Z \rightarrow Y, \mu: Y \rightarrow Q$, $\theta: Q \rightarrow X$ they have a resultant $\theta \mu k: Z \rightarrow X$. I say that $\theta \mu k$ is an $(N-1)$ homotopy inverse of $i: X \rightarrow Z$. For let $K$ be a finite polyhedron of at most $N-1$ dimensions and let $\phi: K \rightarrow X$ be a given map. Then $\mu k i \phi$ maps $K$ into $Q$ and is homotopic to a map, $\phi^{\prime}: K \rightarrow Q$, such that $\phi^{\prime} K \subset Q^{N-1}$. Since $i \theta\left|Q^{N-1} \simeq j \mu^{\prime}\right| Q^{N-1}$ it follows that $i \theta \phi^{\prime} \simeq j \mu^{\prime} \phi^{\prime}$. Therefore

$$
i \theta \mu k i \phi \simeq i \theta \boldsymbol{\phi}^{\prime} \simeq j \mu^{\prime} \boldsymbol{\phi}^{\prime} \simeq j \mu^{\prime} \mu k i \phi .
$$

Since $\mu^{\prime} \mu \simeq 1, j k \simeq 1$ it follows that

$$
i \theta \mu k i \phi \simeq j k i \phi \simeq i \phi
$$

and hence that $\theta \mu k i \phi \simeq \phi$, according to Lemma 6 below. Therefore $\theta \mu k i \simeq_{N-1} 1$. A similar but rather simpler argument shows that $i \theta \mu k \simeq_{N-1} 1$. Therefore $i$, and hence $f: X \rightarrow Y$, is an $(N-1)$-homotopy equivalence.

Conversely ${ }^{14}$ let $f: X \rightarrow Y$ have an $(N-1)$-homotopy inverse,

${ }^{13}$ If $\operatorname{dim}(Z-X)<\Delta X+1$ this is weaker than Hu's version.

${ }^{14}$ This is nontrivial because of questions concerning the base point. For example, let $u, u^{\prime}:\left(S^{n}, p_{0}\right) \rightarrow\left(X, x_{0}\right)$ be maps representing two given elements of $\pi_{n}(X)$ $=\pi_{n}\left(X, x_{0}\right)$. Assume that $g f x_{0}=x_{0}$. Then the images of $p_{0}$ will, in general, describe circuits, which represent different elements of $\pi_{1}\left(X, x_{0}\right)$, in the homotopies $g f u \simeq u$, $g f u^{\prime} \sim u^{\prime}$. 
$g: Y \rightarrow X$. Let vertices $p_{0} \in P^{0}, q_{0} \in Q^{0}$ be chosen as base points for all the groups $\pi_{n}(P), \pi_{n}(Q)$ and let $x_{0}=\lambda^{\prime} p_{0}, y_{0}=\mu^{\prime} q_{0}$ be taken as base points for $\pi_{n}(X), \pi_{n}(Y)$. After suitable homotopies of $\lambda, \mu$, if necessary, we assume that $\lambda x_{0}=p_{0}, \mu y_{0}=q_{0}$. We then deform $f$ so that $f x_{0}=y_{0}$ and finally $g$ so that $g y_{0}=x_{0}$. Let $g_{n}: \pi_{n}(Y) \rightarrow \pi_{n}(X)$ be the homomorphism induced by $g$ and let $\lambda_{n}, \mu_{n}, \lambda_{n}^{\prime}, \mu_{n}^{\prime}$ be the homomorphisms of $\pi_{n}(X), \pi_{n}(Y), \pi_{n}(P), \pi_{n}(Q)$, which are induced by $\lambda, \mu, \lambda^{\prime}, \mu^{\prime}$. Since $\lambda^{\prime} \lambda \simeq 1, \mu^{\prime} \mu \simeq 1$ we have $\lambda_{n}^{\prime} \lambda_{n}=\alpha_{n}, \mu_{n}^{\prime} \mu_{n}=\beta_{n}$, where $\alpha_{n}, \beta_{n}$ are automorphisms ${ }^{15}$ of $\pi_{n}(X), \pi_{n}(Y)$. Also $g f \lambda^{\prime}\left|P^{N-1} \simeq \lambda^{\prime}\right| P^{N-1}$, whence $g_{n} f_{n} \lambda_{n}^{\prime}=\alpha_{n}^{\prime} \lambda_{n}^{\prime}$ if $1 \leqq n \leqq N-1$, where $\alpha_{n}^{\prime}: \pi_{n}(X) \rightarrow \pi_{n}(X)$ is an automorphism. Therefore

$$
\begin{aligned}
g_{n} f_{n} \alpha_{n} & =g_{n} f_{n} \lambda_{n}^{\prime} \lambda_{n}=\alpha_{n}^{\prime} \lambda_{n}^{\prime} \lambda_{n} \\
& =\alpha_{n}^{\prime} \alpha_{n},
\end{aligned}
$$

whence $g_{n} f_{n}=\alpha_{n}^{\prime}$. Similarly $f_{n} g_{n}=\beta_{n}^{\prime}$ where $\beta_{n}^{\prime}: \pi_{n}(Y) \rightarrow \pi_{n}(Y)$ is an automorphism. Hence ${ }^{16}$ it easily follows that $f_{n}$ is an isomorphism onto and the theorem is established.

5. Lemmas on homology. Let $A$ and $B \subset A$ be any arcwise connected spaces. Then we have:

LEMMA 2. If the injection homomorphism $i_{1}: \pi_{1}(B) \rightarrow \pi_{1}(A)$ is an isomorphism (into) then $\pi_{2}(A, B)$ is Abelian.

Consider the homotopy sequence

$$
\pi_{2}(A) \stackrel{1}{\rightarrow} \pi_{2}(A, B) \stackrel{2}{\rightarrow} \pi_{1}(B) \stackrel{3}{\rightarrow} \pi_{1}(A) .
$$

If (3) is an isomorphism, then (2) is into zero and (1) is onto. Therefore the lemma follows from the fact that $\pi_{2}(A)$ is Abelian.

LEMMA 3. If $\pi_{1}(A)=1$ then the natural homomorphism $\pi_{2}(A, B)$ $\rightarrow \mathrm{H}_{2}(A, B)$ is onto and its kernel is the commutator subgroup of $\pi_{2}(A, B)$. If also $\pi_{1}(B)=1$ and $H_{r}(A, B)=0$ for $r=1,2, \cdots, n-1$ $(n \geqq 2)$, then the natural homomorphism $\pi_{n}(A, B) \rightarrow H_{n}(A, B)$ is an isomorphism onto.

This is an extension of a theorem due to W. Hurewicz, to which it reduces in case $B$ is a single point. S. Eilenberg [12, p. 443] has given a proof of Hurewicz's theorem which, with minor modifications, establishes Lemma 3.

On combining Lemmas 2 and 3 we have the following lemma.

\footnotetext{
${ }^{15}$ These are inner automorphisms if $n=1$ and are due to the operators in $\pi_{1}(X)$, $\pi_{1}(Y)$ if $n>1$.

${ }^{16}$ Cf. (7.4) below.
} 
LEMMA 4. If $\pi_{1}(A)=1, \pi_{1}(B)=1, H_{n}(A, B)=0$ for every value of $n$, then $\pi_{n}(A, B)=0$ for each $n$.

6. Proof of Theorem 3. Let $f: X \rightarrow Y$ be a map which induces isomorphisms of $\pi_{1}(X)$ and $H_{n}(\tilde{X})(n=0,1, \cdots)$ on to $\pi_{1}(Y)$ and $H_{n}(\tilde{Y})$ and let the notation be the same as in $\$ 3$. Let $Z$ be the universal covering space of $Z$, with $x_{0} \in X$ as base point. Since $f_{1}: \pi_{1}(X)$ $\rightarrow \pi_{1}(Y)$ is an isomorphism onto it follows from an argument used at the beginning of $\S 3$ that $i_{1}: \pi_{1}(X) \rightarrow \pi_{1}(Z)$ is an isomorphism onto. Therefore we may identify $\tilde{X}$, and similarly $\tilde{Y}$, with the sub-sets of $\tilde{Z}$ which cover $X$ and $Y$ respectively. Then it follows from arguments similar to those at the beginning of $\$ 3$, including (3.1), with $X, Y, Z$ replaced by $\tilde{X}, \tilde{Y}, \tilde{Z}$ and homotopy groups replaced by homology groups, that all the relative homology groups $H_{n}(\tilde{Z}, \tilde{X})(n=1,2, \cdots)$ are zero. Since $\pi_{1}(\tilde{X})=1, \pi_{1}(\tilde{Z})=1$ it follows from Lemma 4 that $\pi_{n}(\tilde{Z}, \tilde{X})=0$ for each $n \geqq 1$. Therefore $\pi_{n}(Z, X)=0(n \geqq 2)$ and $i_{1}: \pi_{1}(X)$ $\rightarrow \pi_{1}(Z)$ is onto. Therefore the theorem follows from the proof of the simpler version of Theorem 1, which was mentioned towards the end of $\$ 3$.

7. Proof of Theorem 4. It follows from the conditions (a), (b) of Theorem 4 and from (3.1), with $X, Y, Z$ replaced by $\tilde{X}, \tilde{Y}, \tilde{Z}$ and homotopy groups replaced by homology groups, that $H_{n}(\tilde{Z}, \tilde{X})=0$ for $n=2, \cdots, N-1$. Therefore $\pi_{n}(Z, X)=0$ and hence $\pi_{n}(Z, X)=0$ for $n=2, \cdots, N-1$. Also $f_{1}$ is onto, whence $\pi_{1}(Z, X)=0$, and $f_{N-1}$ is an isomorphism. Therefore the first half of Theorem 4 follows from the proof of Theorem 2 .

The second half of Theorem 4 is trivial if $N=2$, since $\tilde{X}$ and $\tilde{Y}$ are simply connected. Therefore we assume that $N \geqq 3$. Using the same notation as before, let $\widetilde{P}$ be the universal covering space of $P$, with a base point $p_{0} \in P^{0}$. As at the end of $\$ 4$, let the base points $x_{0} \in X$, $y_{0} \in Y, q_{0} \in Q^{0}$ be such that

$$
x_{0}=\lambda^{\prime} p_{0}=g y_{0}, \quad y_{0}=\mu^{\prime} q_{0}=f x_{0},
$$

and let $\tilde{x}_{0}, \tilde{p}_{0}$, etc., be the base points in $\tilde{X}, \tilde{P}$, etc. Let $\tilde{\lambda}^{\prime}: \widetilde{P} \rightarrow \tilde{X}$ be the map which covers $\lambda^{\prime}: P \rightarrow X$, meaning that $\tilde{\lambda}^{\prime} \tilde{p}_{0}=\tilde{x}_{0}$ and $\lambda^{\prime} p=p \bar{\lambda}^{\prime}$, where $p$ denotes both projections $p: \widetilde{P} \rightarrow P, p: \widetilde{X} \rightarrow X$. According to a remark in $\$ 1$ the map $\bar{\lambda}^{\prime}$ has a right homotopy inverse, $\tilde{\lambda}: \tilde{X} \rightarrow \tilde{P}$.

Let $h_{0}, h_{1}: \tilde{X} \rightarrow A$ be maps of $\tilde{X}$ in any space, $A$, and let

$$
h_{0} \tilde{\lambda}^{\prime}\left|\tilde{P}^{N-1} \simeq h_{1} \tilde{\lambda}^{\prime}\right| \tilde{P}^{N-1}
$$

where $\tilde{P}^{n}$ is the $n$-section of $\tilde{P}$. Let $\phi: K \rightarrow \tilde{X}$ be a given map, where 
$K$ is a polyhedron and $\operatorname{dim} K \leqq N-1$. Then $\bar{\lambda} \phi: K \rightarrow \widetilde{P}$ is homotopic to a map, $\phi^{\prime}$, such that $\phi^{\prime} K \subset \widetilde{P}^{N-1}$. Since $\bar{\lambda}^{\prime} \bar{\lambda} \simeq 1$ we have $h_{i} \phi \simeq h_{i} \bar{\lambda}^{\prime} \bar{\lambda} \phi$ $\simeq h_{i} \tilde{\lambda}^{\prime} \phi^{\prime}(i=0,1)$. Since $\phi^{\prime} K \subset \widetilde{P}^{N-1}$ it follows from (7.2) that $h_{0} \bar{\lambda}^{\prime} \phi^{\prime}$ $\simeq h_{1} \tilde{\lambda}^{\prime} \phi^{\prime}$ and hence that $h_{0} \phi \simeq h_{1} \phi$. Therefore

$$
h_{0} \simeq_{N-1} h_{1}
$$

in consequence of (7.2).

Let $f: X \rightarrow Y$ and $g: Y \rightarrow X$ be such that $g f \simeq_{N-1} 1, f g \simeq_{N-1} 1$ and let $\tilde{f}: \tilde{X} \rightarrow \widetilde{Y}, \tilde{g}: \widetilde{Y} \rightarrow \tilde{X}$ be the maps which cover $f, g$. Since $g f \simeq_{N-1} 1$ there is a homotopy, $\theta_{t}: P^{N-1} \rightarrow X$, such that $\theta_{0}=g f \lambda^{\prime}\left|P^{N-1}, \theta_{1}=\lambda^{\prime}\right| P^{N-1}$. It follows from (7.1) that $\theta_{0} p_{0}=\theta_{1} p_{0}=x_{0}$. Let $\theta_{t}$ be lifted into the homotopy, $\tilde{\theta}_{t}: \widetilde{P}^{N-1} \rightarrow \tilde{X}$, such that $\tilde{\theta}_{0} \tilde{p}_{0}=\tilde{x}_{0}, \theta_{t} p=p \tilde{\theta}_{t}$. It follows from (7.1) that $\tilde{\theta}_{0}=\tilde{g} \tilde{f} \bar{\lambda}^{\prime} \mid \tilde{P}^{N-1}$ and that $\tilde{\theta}_{1}=u \tilde{\lambda}^{\prime} \mid \tilde{P}^{N-1}$, where $u: \tilde{X} \rightarrow \tilde{X}$ is a transformation in the covering group. Hence it follows from (7.2) and (7.3), with $A=\tilde{X}, h_{0}=\tilde{g} \tilde{f}, h_{1}=u$, that $\tilde{g} \tilde{f} \simeq_{N-1} u$. Similarly $\tilde{f} \tilde{g}$ $\simeq_{N-1} v$, where $v: \tilde{Y} \rightarrow \tilde{Y}$ is in the group of covering transformations. Let $\bar{g}=u^{-1} \tilde{g}$. Then $\bar{g} \tilde{f} \simeq_{N-1} 1$ and

$$
\begin{aligned}
\tilde{f} \bar{g} & =\left(v^{-1} v\right) \tilde{f} \bar{g} \simeq_{N-1}\left(v^{-1} \tilde{f} \tilde{g}\right) \tilde{f} \bar{g} \\
& =v^{-1} \tilde{f}(\tilde{g} \tilde{f}) \bar{g} \simeq_{N-1} v^{-1} \tilde{f} u \bar{g} \\
& =v^{-1} \tilde{f} \tilde{g} \simeq_{N-1} 1 .
\end{aligned}
$$

Therefore $\tilde{f}$ is an $(N-1)$-homotopy equivalence.

Since $\bar{\lambda}^{\prime} \bar{\lambda} \simeq 1$ it follows that any (singular) cycle in $X^{z}$ is homologous to a continuous ${ }^{17}$ cycle. Similarly any cycle in $\tilde{Y}$ is homologous to a continuous cycle. Therefore the homomorphism $H_{n}(\tilde{X}) \rightarrow H_{n}(\tilde{Y})$, which is induced by $\bar{f}$, is obviously an isomorphism onto if $n \leqq N-1$. This completes the proof.

8. Two lemmas. Let $e^{n}(n \geqq 1)$ be an $n$-cell, which is an open subset of a Hausdorff space, $A$, and let $A_{0}=A-e^{n}$. Let $\bar{e}^{n}$, the closure of $e^{n}$, be the image of an $n$-simplex, $\sigma^{n}$, in a map, $\phi:\left(\sigma^{n}, \dot{\sigma}^{n}\right) \rightarrow\left(\bar{e}^{n}, \bar{e}^{n} \cap A_{0}\right)$, such that $\phi \mid\left(\sigma^{n}-\dot{\sigma}^{n}\right)$ is a homeomorphism onto $e^{n}$. Let $B$ and $B_{0} C B$ be arcwise connected spaces such that $\pi_{n}\left(B, B_{0}\right)=0$ and let $f_{0}:\left(A, A_{0}\right)$ $\rightarrow\left(B, B_{0}\right)$ be a given map.

Lemma 5. There is a homotopy, $f_{t}:\left(A, A_{0}\right) \rightarrow\left(B, B_{0}\right)$, rel. $A_{0}$, such that $f_{1} A \subset A_{0}$.

Since $\pi_{n}\left(B, B_{0}\right)=0$ there is a homotopy, $\psi_{t}:\left(\sigma^{n}, \dot{\sigma}^{n}\right) \rightarrow\left(B, B_{0}\right)$, rel.

${ }^{17}$ For example, to the image under $\tilde{\lambda}^{\prime}$ of a continuous cycle in $\widetilde{P}$. By a continuous, $n$-dimensional cycle is meant the image in a map, $K^{n} \rightarrow \widetilde{X}$, of a cycle carried by an $n$-dimensional complex $K^{n}$. 
$\dot{\sigma}^{n}$, such that $\psi_{0} p=f_{0} \phi p\left(p \in \sigma^{n}\right), \psi_{1} \sigma^{n} \subset B_{0}$. Let $g_{t}: \bar{e}^{n} \rightarrow B$ be given by $g_{t}=\psi_{t} \phi^{-1}$. Clearly $\phi^{-1} e^{n}$, and hence $g_{t} \mid e^{n}$, is single-valued. If $a \in \bar{e}^{n}-e^{n}$ $=\bar{e}^{n} \cap A_{0}$, then $\phi^{-1} a \subset \dot{\sigma}^{n}$ and $g_{t} a=\psi_{0} \phi^{-1} a=f_{0} \phi \phi^{-1} a=f_{0} a$. Therefore $g_{t}$ is single-valued and hence continuous (see $[8, \S 5])$. Moreover $g_{t}\left|\bar{e}^{n} \cap A_{0}=f_{0}\right| \bar{e}^{n} \cap A_{0}$. Therefore the requirements of the lemma are satisfied by $f_{t}$, which is given by $f_{t}\left|A_{0}=f_{0}, f_{t}\right| \bar{e}^{n}=g_{t}$.

Let $A$ be a closed subset of a separable metric space, $A^{\prime}$, let $B$ be a separable metric ANR and let $f_{0}: A \rightarrow B$ have an extension $f_{0}^{\prime}: A^{\prime}$ $\rightarrow B$. Then the homotopy, $f_{t}$, of Lemma 5 can be extended to a homotopy $f_{t}^{\prime}: A^{\prime} \rightarrow B$.

Now let $B$ be a separable, metric ANR and let the homomorphism, $i_{n}: \pi_{n}\left(B_{0}\right) \rightarrow \pi_{n}(B)$, which is induced by the identical map $i: B_{0} \rightarrow B$, be an isomorphism onto for $n=1, \cdots, m$. Then it follows from (3.1), with $X, Z$ replaced by $B_{0}, B$, that $\pi_{n}(Z, X)=0$ for $n=1, \cdots, m$. Let $f_{0}, f_{1}: K^{m} \rightarrow B_{0}$ be maps of an $m$-dimensional, simplicial complex, $K^{m}$, into $B_{0}$. Then we have:

\section{LEMma 6. If if $\simeq$ if $f_{1}($ in $B)$ then $f_{0} \simeq f_{1}\left(\right.$ in $\left.B_{0}\right)$.}

Let $g_{t}: K^{m} \rightarrow B$ be a deformation of $g_{0}=i f_{0}$ into $g_{1}=i f_{1}$ and let $g: K^{m} \times I \rightarrow B$ be given by $g(x, t)=g_{t} x\left(x \in K^{m}\right)$. Let $C_{n}=\left(K^{m} \times 0\right)$ $\cup\left(K^{n-1} \times I\right) \cup\left(K^{m} \times 1\right)$. Since $\pi_{n}\left(B, B_{0}\right)=0 \quad(n=1, \cdots, m)$ it follows from repeated applications of Lemma 5 , with $\bar{e}^{n}=\sigma_{i}^{n-1} \times I$, where $\sigma_{1}^{n-1}, \sigma_{2}^{n-1}, \ldots$ are the $(n-1)$-simplexes in $K^{m}$, that $g$ is homotopic, rel. $\left(K^{m} \times 0\right) \cup\left(K^{m} \times 1\right)$, to a map $g^{\prime}: K^{m} \times I \rightarrow B$, such that $g^{\prime} C_{m} \subset B_{0}$. Let $E_{i}^{m+1}=\sigma_{i}^{m} \times I(i=1,2, \cdots)$. Then $g^{\prime} \dot{E}_{i}^{m+1} \subset B_{0}$ and $g^{\prime} \mid \dot{E}_{i}^{m+1}$ is contractible in $B$. Since $i_{m}: \pi_{m}\left(B_{0}\right) \rightarrow \pi_{m}(B)$ is an isomorphism it follows that $g^{\prime} \mid \dot{E}_{i}^{m+1}$ is contractible in $B_{0}$. Therefore there is a map $h_{i}: E^{m+1} \rightarrow B_{0}$ such that $h_{i} p=g^{\prime} p$ if $p \in \dot{E}_{i}^{m+1}$. Let $f: K^{m} \times I \rightarrow B_{0}$ be given by $f p=g^{\prime} p$ if $p \in C_{m}, f p=h_{i} p$ if $p \in E_{i}^{m+1}$. Then $f_{t}: K^{m} \rightarrow B_{0}$, given by $f_{t} x=f(x, t)$, is a homotopy of $f_{0}$ into $f_{1}$, which completes the proof.

\section{REFERENCES}

1. R. H. Fox, Ann. of Math. vol. 44 (1943) pp. 40-50.

2. —- Ann. of Math. vol. 42 (1941) pp. 333-370.

3. H. Samelson, Ann. of Math. vol. 45 (1944) pp. 448-449.

4. S. Lefschetz, Algebraic topology, Amer. Math. Soc. Colloquium Publications vol. 27, New York, 1942. 1942.

5. - Topics in topology, Annals of Mathematics Studies, No. 10, Princeton,

6. J. H. C. Whitehead, Proc. London Math. Soc. vol. 45 (1939) pp. 243-327.

7. - Ann. of Math. vol. 42 (1941) pp. 1197-1239.

8. - Note on a theorem due to Borsuk, Bull. Amer. Math. Soc. vol. 54 (1948) pp. 1125-1132. 
9. Sze-Tsen Hu, Proc. Cambridge Philos. Soc. vol. 43 (1947) pp. 314-320.

10. Hsein-Chung Wang, Proceedings Akademie van Wetenschappen, Amsterdam, vol. 50 (1947).

11. K. Reidemeister, Topologie der Polyeder, Leipzig, 1933.

12. S. Eilenberg, Ann. of Math. vol. 45 (1944) pp. 407-447.

13. - Fund. Math. vol. 32 (1939) pp. 167-175.

14. W. Hurewicz, Proceedings Akademie von Wetenschappen, Amsterdam, vol. 38 (1935) pp. 521-528.

15. K. Borsuk, Fund. Math. vol. 24 (1935) pp. 249-558.

Magdalen College, Oxford University

\section{TOPOLOGICAL CHARACTERIZATION OF FIELDS WITH VALUATIONS}

\section{DANIEL ZELINSKY ${ }^{1}$}

1. Introduction. A topological field is a (commutative) field which is also a topological space satisfying the separation axiom $T_{0}$, and in which addition, subtraction and multiplication are continuous, twovariable functions. For our purposes it is unnecessary to assume that division is continuous.

If $F$ is any field, topological or not, we define a (nonarchimedean) valuation of $F$ to be a function $v$ on $F$ to a linearly ordered group $\Gamma$ with the symbol 0 adjoined, such that

$$
\begin{aligned}
v(x y) & =v(x) v(y), \\
v(x+y) & \leqq \max [v(x), v(y)], \\
v(x) & =0 \text { if and only if } x=0,
\end{aligned}
$$

for all $x, y$ of $F$. It is understood that for every $\gamma$ of $\Gamma, 0<\gamma$ and $0 \gamma=\gamma 0$ $=0$. Such a valuation of a field defines a topology, with respect to which $F$ is a topological field, when we specify that the neighborhoods of 0 in $F$ are the sets $U(\gamma)=[x \in F \mid v(x)<\gamma]$, one for each $\gamma$ in $\Gamma$. If $F$ was a topological field to begin with and the topology defined by the valuation is the same as the original topology of $F$, we say that the valuation preserves the topology of $F$.

The question we intend to answer is, "Which topological fields have valuations preserving their topologies?"

Presented to the Society, April 17, 1948; received by the editors January 21, 1948.

${ }_{1}^{1}$ This paper was written while the author was a National Research Fellow in Mathematics. 